PROCEEDINGS OF THE

AMERICAN MATHEMATICAL SOCIETY

Volume 129, Number 3, Pages 695-698

S 0002-9939(00)05625-

Article electronically published on September 20, 2000

\title{
ALMOST SPLIT SEQUENCES IN CATEGORIES OF REPRESENTATIONS OF QUIVERS
}

\author{
SVERRE O. SMAL $\varnothing$
}

(Communicated by Ken Goodearl)

\begin{abstract}
Let $k$ be a field and $Q$ a connected quiver. In this note it is proved that the category of finite dimensional representations of $Q$ over $k$ has almost split sequences if and only if $Q$ either is without oriented cycles or consists of a single oriented cycle.
\end{abstract}

\section{INTRODUCTION}

The aim of this note is to prove the following result.

Theorem 1. Let $Q$ be a connected finite quiver, $k$ a field and $\operatorname{fd}(Q, k)$ the category of finite dimensional representations of $Q$ over $k$. Then $\operatorname{fd}(Q, k)$ has almost split sequences if and only if one of the following two conditions is satisfied:

(i) $Q$ contains no oriented cycles or

(ii) $Q$ is $\widetilde{A}_{n}$ for some $n$ with cyclic orientation.

Let $\mathcal{C}$ be an abelian Krull-Schmidt-category, i.e. the indecomposable objects in $\mathcal{C}$ have local endomorphism rings and each object in $\mathcal{C}$ can be decomposed as a finite direct sum of indecomposables, and then in a unique way up to isomorphism by the Krull-Schmidt theorem.

The category $\mathcal{C}$ is said to have right almost split morphisms if for each indecomposable object $X$ in $\mathcal{C}$, there is a morphism $f: Y \rightarrow X$ in $\mathcal{C}$ such that the cokernel, $\operatorname{Coker}(, f)$, of the natural morphism $\operatorname{Hom}_{\mathcal{C}}(, f)$ as a functor on $\mathcal{C}$ takes the value 0 for all indecomposable objects $Y$ not isomorphic to $X$ and the value $\operatorname{Hom}_{\mathcal{C}}\left(X^{\prime}, X\right) / \operatorname{rad}\left(X^{\prime}, X\right)$ for the indecomposable objects $X^{\prime}$ isomorphic to $X$. Here $\operatorname{rad}\left(X^{\prime}, X\right)$ is the set of nonisomorphisms from $X^{\prime}$ to $X$ which is a subgroup of $\operatorname{Hom}\left(X^{\prime}, X\right)$ since the endomorphism ring of $X$ is local. The morphisms are sent to their residues. A morphism $f: Y \rightarrow X$ satisfying the above property is called a right almost split morphism in $\mathcal{C}$.

Dually, $\mathcal{C}$ is said to have left almost split morphisms if for each indecomposable object $X$ in $\mathcal{C}$, there is a morphism $f: X \rightarrow Y$ in $\mathcal{C}$ such that the cokernel, $\operatorname{Coker}(f$,$) , of the natural morphism \operatorname{Hom}_{\mathcal{C}}(f$,$) satisfies the same condition$ as above. That is, $\operatorname{Coker}(f$,$) applied to an indecomposable object Y$ in $\mathcal{C}$ is $\operatorname{Hom}(X, Y) / \operatorname{rad}(X, Y)$ where $\operatorname{rad}(X, Y)$ is the subgroup of nonisomorphisms from $X$ to $Y$. A morphism $f: X \rightarrow Y$ satisfying the above property is called a left almost split morphism in $\mathcal{C}$.

Received by the editors April 20, 1999 and, in revised form, June 11, 1999.

1991 Mathematics Subject Classification. Primary 16E10, 16G10, 16G20, 16P10. 
The category $\mathcal{C}$ is said to have almost split morphisms if it has both right and left almost split morphisms. It is said to have almost split sequences if, in addition, for each indecomposable nonprojective module $X$ in $\mathcal{C}$ there exists an exact sequence $0 \rightarrow Z \rightarrow Y \rightarrow X \rightarrow 0$ with $Z$ indecomposable and where $Z \rightarrow Y$ is left almost split in $\mathcal{C}$ and where $Y \rightarrow X$ is right almost split in $\mathcal{C}$; and for each indecomposable noninjective module $Z$ in $\mathcal{C}$ there exists an exact sequence $0 \rightarrow Z \rightarrow Y \rightarrow X \rightarrow 0$ with $X$ indecomposable and where $Z \rightarrow Y$ is left almost split in $\mathcal{C}$ and where $Y \rightarrow X$ is right almost split in $\mathcal{C}$.

For background on the representation theory of artin algebras including finite dimensional algebras, and on the theory of representations of quivers, the reader is referred to the book ARS.

A celebrated result of Auslander and Reiten is the following theorem (see [ARS]).

Theorem 2. If $\Lambda$ is an artin algebra, then the category of finitely generated left $\Lambda$-modules has both left and right almost split morphisms as well as almost split sequences.

\section{PROOF}

Now to the proof of the result of this note.

Let us start by giving the arguments that if (i) or (ii) is satisfied, then $\operatorname{fd}(Q, k)$ has almost split sequences.

If (i) is satisfied, then $\operatorname{fd}(Q, k)$ is equivalent to the category of finitely generated left $k Q$-modules where $k Q$ is the path algebra of $Q$ over $k$, which is then a finite dimensional hereditary algebra. Hence the theorem of Auslander and Reiten gives the existence of almost split sequences. Therefore we only have to consider the situation described in (ii).

If $Q$ is $\widetilde{A}_{n}$ for some $n$, with cyclic orientation, then $\operatorname{fd}(Q, k)$ is an abelian finite length subcategory, closed with respect to extensions, within the category of all representations of $Q$ over $k$. The category of all representations of $Q$ over $k$ is again equivalent to the category of all left modules over $k Q$, the path algebra of $Q$ over $k$, which is still an hereditary algebra. Hence, $\operatorname{fd}(Q, k)$ is a finite length hereditary abelian category. Number the vertices in $Q$ as $0,1, \ldots, n$ with an arrow from $i$ to $i+1$ for $i=0,1, \ldots, n-1$ and an arrow from $n$ to 0 . This can be expressed by saying that there is an arrow from $i$ to $j$ if $n+1$ divides $j-1-i$, and just one loop at 0 if $n=0$. We will now describe the simple objects of $\operatorname{fd}(Q, k)$.

There are the "discrete" simples $S_{i}$, one for each vertex $i$ of $Q$, where at vertex $i$ there is a one dimensional vector space and all morphisms and other spaces are zero.

Next let $(V, f)$ be a finite dimensional representation of $Q$ over $k$, such that $f_{i}: V_{i} \rightarrow V_{(i+1)}$ is not a monomorphism, where $(i+1)$ denotes the residue of $i+1$ modulo $n+1$. Then $S_{i}$ is a subrepresentation and therefore $(V, f)$ is simple only if it is isomorphic to $S_{i}$. In the same way, if $f_{i}$ is not an epimorphism, then $S_{(i+1)}$ is a quotient representation of $(V, f)$, and hence $(V, f)$ is simple only if $(V, f)$ is isomorphic to $S_{(i+1)}$. Hence the other possibilities for simple representations are the representations where all $f_{i}$ are isomorphisms. Then using the $n$ first isomorphisms as identifications, one obtains a $k[X]$-module, and the lattice of submodules of this $k[X]$-module is exactly the same as the lattice of subrepresentations of $(V, f)$. This gives rise to the set of "continuous" simple representations, one for each irreducible 
monic polynomial in $k[X]$ except the polynomial $X$. A convenient way of representing such a simple $S_{g}$, corresponding to an irreducible monic polynomial $g \neq X$, is by considering the space of polynomials $k\left[X^{n+1}\right]$ in $X^{n+1}$ and letting the vector space at vertex $i$ be the space of polynomials $X^{i} k\left[X^{n+1}\right]$ modulo the subspace given by the ideal generated by $g\left(X^{n+1}\right)$ multiplied by $X^{i}$, and letting all maps be induced by multiplication by $X$.

The projective representation $P_{0}$ corresponding to vertex 0 can also be described by using the space $k\left[X^{n+1}\right]$. In vertex $i$ let the space be the space of polynomials $X^{i} k\left[X^{n+1}\right]$ for $i=0, \ldots, n$ and all the maps be multiplication by $X$. The projective representation corresponding to vertex $i$ is now isomorphic to the subrepresentation of $P_{0}$ generated by $X^{i}$ in vertex $i$. It is then easy to write down projective presentations of the simple representations. They are

$$
0 \rightarrow P_{(j-1)} \rightarrow P_{j} \rightarrow S_{j} \rightarrow 0
$$

for the discrete simple representation $S_{j}$, and

$$
0 \rightarrow P_{0} \rightarrow P_{0} \rightarrow S_{g} \rightarrow O
$$

where the map from $P_{0}$ to $P_{0}$ is just multiplication with $g\left(X^{n+1}\right)$ in each vertex for the continuous simple representations corresponding to the monic irreducible polynomial $g \neq X$. Observe that the dimension of $\operatorname{Hom}_{\mathrm{fd}(Q, k)}\left(P_{i}, S_{g}\right)$ as a $k$-space is the degree of $g$ for all the projective representations $P_{i}$ and each continuous simple representation $S_{g}$, and that the endomorphism ring of $S_{g}$ is the field extension $k[X] /(g)$. Using these resolutions one obtains the following properties. For the discrete simple representations one has $\operatorname{Ext}\left(S_{i}, S_{j}\right) \neq 0$ only when $n+1$ divides $j-1-i$ and if this Ext-group is nonzero, it is one dimensional over $k$. For each continuous simple representation $S_{g}$, one obtains $\operatorname{Ext}\left(S_{g}, S_{j}\right)=0$ for all discrete simple representations $S_{j}$, and $\operatorname{Ext}\left(S_{g}, S_{h}\right)=0$ and all continuous simple representations $S_{h}$ except when $h=g$. For $g=h$ this Ext-group is a one-dimensional space over the endomorphism ring of $S_{g}$. In the same way one obtains $\operatorname{Ext}\left(S_{j}, S_{g}\right)=0$ for a monic irreducible polynomial $g \neq X$ and discrete simple $S_{j}$. From this one finds that the category $\operatorname{fd}(Q, k)$ in this case is the coproduct of finite length uniserial hereditary abelian categories indexed over the monic irreducible polynomials over $k$. For the irreducible polynomial $X$ there are $n+1$ isomorphism classes of simple representations, the discrete simple representations, and for each of the monic irreducible polynomials $g \neq X$, there is one simple representation. Hence, one can index the indecomposable modules with $(\{0, \ldots, n\} \cup\{g \mid g \neq X$ is a monic irreducible polynomial in $k[X]\}) \times \mathbb{N}$. The modules indexed by $\{0, \ldots, n\} \times \mathbb{N}$ correspond to part of what is called string modules for special biserial algebras. Each of the indecomposable objects $A$ in this category has the property that $\operatorname{Ext}^{1}(A) \neq$,0 and $\operatorname{Ext}^{1}(, A) \neq 0$. From this information it is possible to construct almost split sequences, and they are given by the following:

$$
0 \rightarrow(g, 1) \rightarrow(g, 2) \rightarrow(g, 1) \rightarrow 0
$$

for each monic irreducible polynomial $g \neq X$ (holds also for $X$ if $n=0$ ), and

$$
0 \rightarrow(g, m) \rightarrow(g, m+1) \amalg(g, m-1) \rightarrow(g, m) \rightarrow 0
$$

for each monic irreducible polynomial $g \neq X$ (holds also for $X$ if $n=0$ ) and $m \geq 2$, and

$$
0 \rightarrow(j, 1) \rightarrow(j, 2) \rightarrow((j-1), 1) \rightarrow 0
$$


and

$$
0 \rightarrow(j, m) \rightarrow(j, m+1) \amalg((j-1), m-1) \rightarrow((j-1), m) \rightarrow 0
$$

for $m \geq 2$ for the uniserial category corresponding to the irreducible polynomial $X$. Here $(q)$ is the residue of $q$ modulo $n+1$.

This finishes one of the claims in Theorem 1.

Now the other part will follow from the following somewhat stronger statement

Proposition 3. If $Q$ is a connected quiver containing $\widetilde{A}_{n}$ with cyclic orientation as a proper subquiver, then $\operatorname{fd}(Q, k)$ has neither left nor right almost split morphisms.

Assume that the quiver $Q$ contains an oriented cycle $\widetilde{A}_{n}$ and an additional arrow $\alpha$ not in $\widetilde{A}_{n}$, ending or starting in a vertex in $\widetilde{A}_{n}$. Let us treat the case in which $\alpha$ ends in a vertex of $\widetilde{A}_{n}$ and let $p$ be the starting vertex of $\alpha$ (this may be any vertex of $Q$, including the vertices in the subquiver $\widetilde{A}_{n}$ ). Then it is clear that the discrete simple at vertex $p$ has extensions with all the continuous simple representations of $\widetilde{A}_{n}$, and hence all the continuous simple representations of $\widetilde{A}_{n}$ would have to be a composition factor of any representation $(W, f)$ with a right almost split morphism $(W, f) \rightarrow S_{p}$, which is impossible for $(W, f)$ in $\operatorname{fd}(Q, k)$. This shows that $\operatorname{fd}(Q, k)$ does not have right almost split morphisms.

To prove that $\mathrm{fd}(Q, k)$ does not have left almost split morphisms, take an extension $(V, f)$ of $S_{p}$ with a fixed continuous simple $S_{g}$ on $\widetilde{A}_{n}$ which fits in a nonsplit exact sequence $0 \rightarrow S_{g} \rightarrow(V, f) \rightarrow S_{p} \rightarrow 0$. This representation $(V, f)$ has extensions $\left(V_{h}, f_{h}\right)$ with all continuous simples $S_{h}$ on $\widetilde{A}_{n}$ with the possible exception of $S_{g}$, such that $\left(V_{h}, f_{h}\right)$ is indecomposable, has Loewy-length equal to 2, with top $S_{p}$ and socle $S_{g} \amalg S_{h}$. From this one deduces that any left almost split morphism $f: S_{g} \rightarrow(W, f)$ has to have the property that the simple representation $S_{p}$ as well as each of the continuous simple representations $S_{h}$ is a composition factor of $(W, f)$ for each monic irreducible polynomial $h$ in $k[X]$. This contradicts that $(W, f)$ is in $\mathrm{fd}(Q, k)$, and hence shows that $\mathrm{fd}(Q, k)$ does not have left almost split morphisms.

We leave to the reader to verify the statement if the additional arrow $\alpha$ starts in a vertex of the subquiver $\widetilde{A}_{n}$.

This finishes the proof of the proposition as well as the proof of the whole theorem.

\section{ACKNowledgement}

This paper was finished during the author's stay at Departemento de Mathematica, Universidad Nacional del Sur, Bahia Blanca, Argentina, and he would like to thank his colleagues there for their hospitality.

\section{REFERENCES}

[ARS] Auslander, M., Reiten, I. and Smalø, S. O. Representation Theory of Artin Algebras, Cambridge University Press, 1995. MR 96c:16015

Institutt for Matematiske fag, NTNU, 7491 Trondheim, Norway

E-mail address: sverresm@math.ntnu.no 